\title{
Spatial Kerr Solitons as Exact Solutions of Maxwell's Equations
}

\author{
Alessandro Ciattoni, Bruno Crosignani, Paolo Di Porto and Amnon Yariv
}

$S_{w}^{p}$ patial Kerr solitons are associated with the optical Kerr effect. The analytic description of this effect is usually framed as the phenomenological approach to optical propagation in continuous media, neglecting contributions to the nonlinear polarizability higher than third-order ones. In this context, it is possible to derive a tensorial analytic expression for the third-order polarizability, which provides a good model of the underlying physical mechanism if the transverse dimensions of the propagating beam exceed some small fraction of the
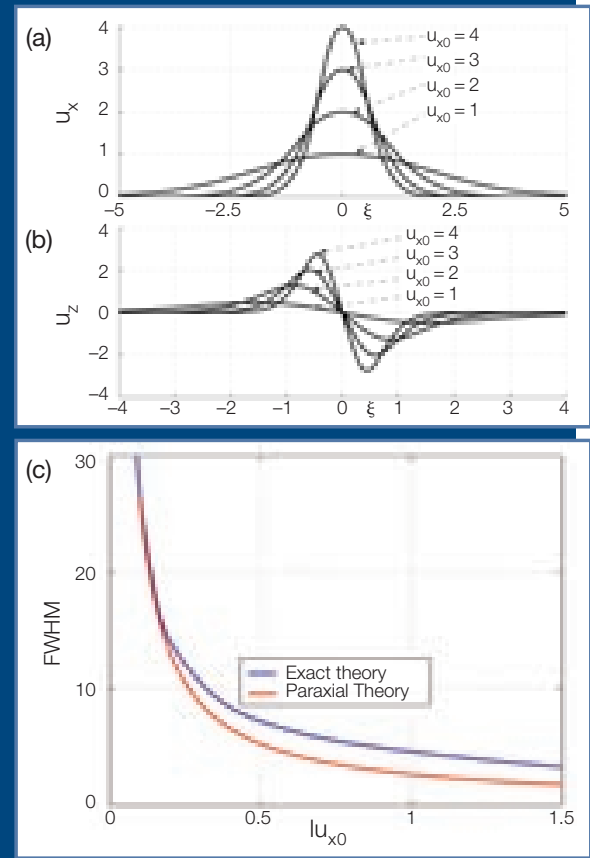

Plot of the transverse (a) and longitudinal (b) field components of various bright solitons. Comparison (c) between the exact (blue line) and paraxial (red line) bright soliton existence curves. Plot of the transverse (d) and longitudinal (e) field components of various dark solitons. Comparison $(f)$ between the exact (blue line) and paraxial (red line) dark soliton existence curves.

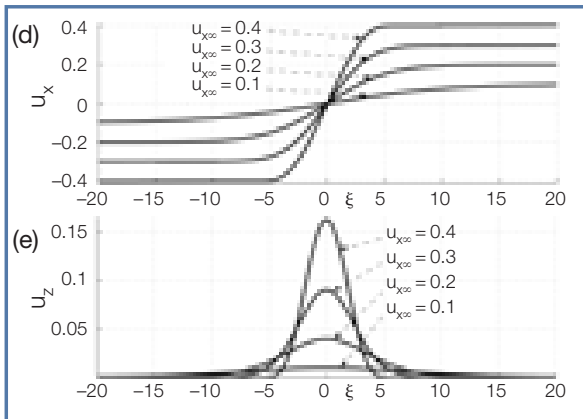

wavelength $\lambda$ and its normalized intensity $u^{2}=\left(n_{2} / n_{0}\right) E^{2}$ is less than unity.

Under these conditions, one typically distinguishes two separate regimes, the paraxial and the non-paraxial one, according to whether the transverse dimension of the beam $\sigma$ is larger or smaller than the wavelength, respectively. In practice, the first situation is usually considered the most relevant one and is dealt with by relying on the non-linear Schroedinger (NLS) equation, which follows from the Helmholtz equation under the paraxial conditions.

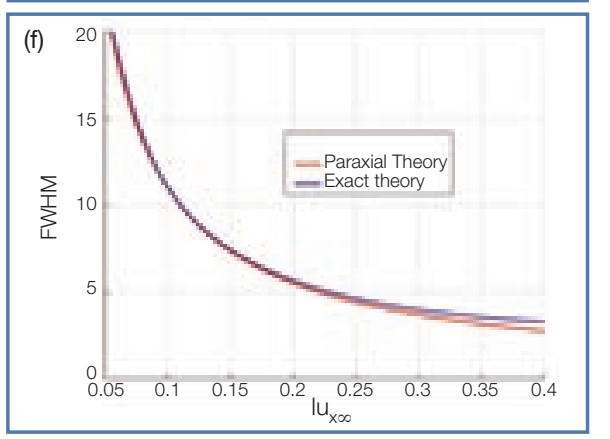

However, non-paraxial effects, which are clearly essential from a fundamental point of view, become significant when subwavelength features are present, and they need to be taken into account in a number of cases. Until recently, this has been done either numerically or analytically, by using some asymptotic expansion in the smallness parameter $\varepsilon=\lambda / \sigma{ }^{1}$
Is it possibile to find $a b$ initio soliton solutions of Maxwell's equations in the presence of the most general expression of the third-order tensorial polarizability describing the optical Kerr effect? We have been able to prove the existence of both bright and dark one-dimensional spatial solitons without resorting to any scalar and paraxial approximation ${ }^{2}$ [see figure, parts (a) (b), (d) and (e)], so that the whole range of possible soliton widths is considered without any formal distinction between paraxial and nonparaxial regimes.

The comparison with the standard paraxial approach reveals, as expected, that, for bright solitons, non-paraxial corrections become relevant whenever the normalized field amplitude $u$ becomes comparable with unity and the associated width $\sigma$ with $\lambda$ [see figure, part (c)]. Dark solitons do not exist if $u^{2}$ exceeds $1 / 6$, so they appear to be much less sensitive to non-paraxial effects within their existence range (f). This is also true in the two-dimensional case, in which solitons are proved to exist as exact vectorial solutions of Maxwell's equations in the form of dark azimuthally polarized solitons, provided that $u^{2}$ is smaller than $1 / 2 .^{3}$

Our results do not simply amount to a refinement of the established optical soliton picture. More fundamentally, they imply that Kerr solitons possess a general significance that extends far beyond the stringent limits of paraxial optics. $\Delta$

[ Alessandro Ciattoni (alessandro.ciattoni@aquila.infn. it) is with the CNR-INFM. Bruno Crosignani and Paolo Di Porto are with the Physics Department of the University of L'Aquila. Amnon Yariv is with the California Institute of Technology, Division of Engineering and Applied Science. ]

\section{References}

1. A. Ciattoni et al. Opt.Lett. 30, 516 (2005).

2. A. Ciattoni et al. J. Opt. Soc. Am. B 22, 1384 (2005).

3. A. Ciattoni et al. Phys. Rev. Lett 94, 073902 (2005). 